\title{
Matrix metalloproteinase-3-dependent generation of a macrophage chemoattractant in a model of herniated disc resorption
}

\author{
Hirotaka Haro, ${ }^{1,2}$ Howard C. Crawford, ${ }^{2}$ Barbara Fingleton, ${ }^{2}$ John R. MacDougall, ${ }^{2}$ \\ Kenichi Shinomiya, ${ }^{3}$ Dan M. Spengler, ${ }^{1}$ and Lynn M. Matrisian ${ }^{2}$ \\ ${ }^{1}$ Department of Orthopaedics and Rehabilitation, and \\ ${ }^{2}$ Department of Cell Biology, Vanderbilt University Medical Center, Nashville, Tennessee 37232, USA \\ ${ }^{3}$ Department of Orthopaedic Surgery, Tokyo Medical and Dental University School of Medicine, Tokyo 113-8519, Japan
}

Address correspondence to: Lynn M. Matrisian, Department of Cell Biology, Vanderbilt University Medical Center, T-2220

MCN, 1161 21st Avenue South, Nashville, Tennessee 37232, USA. Phone: (615) 343-3413; Fax: (615) 343-4539;

E-mail: lynn.matrisian@mcmail.vanderbilt.edu.

Received for publication April 14, 1999, and accepted in revised form December 14, 1999.

\begin{abstract}
Herniated disc (HD) is a common health problem that is resolved by surgery unless spontaneous resorption occurs. HD tissue contains abundant macrophage infiltration and high levels of matrix metalloproteinases (MMPs) MMP-3 and MMP-7. We developed a model system in which disc tissue or isolated chondrocytes from wild-type or MMP-null mice were cocultured with peritoneal macrophages and used this system to investigate the role of MMPs and chondrocyte/macrophage interactions in disc resorption. We observed a marked enhancement of MMP-3 protein and mRNA in chondrocytes after exposure to macrophages. Chondrocytic MMP-3, but not MMP-7, was required for disc resorption, as determined by assaying for a reduction in wet weight and proteoglycan content after 3 days of coculture. Surprisingly, chondrocyte MMP-3 was required for the generation of a macrophage chemoattractant and the subsequent infiltration of the disc tissue by proteolytically active macrophages. We conclude that macrophage induction of chondrocyte MMP-3 plays a major role in disc resorption by mechanisms that include the generation of a bioactive macrophage chemoattractant.
\end{abstract}

J. Clin. Invest. 105:133-141 (2000).

\section{Introduction}

The lifetime prevalence of low back pain (LBP) ranges from 60 to $80 \%$ with an annual incidence of $5 \%(1,2)$. Back complaints are the leading cause for all visits to orthopedic surgeons and neurosurgeons, and herniated discs (HD) are responsible for symptoms in up to $40 \%$ of all patients with $\operatorname{LBP}(1,3,4)$. Symptomatic HD are so pervasive that almost 160 of 100,000 adults in the United States undergo operations for HD every year (5). Therefore, it is important to understand the natural course and pathophysiology of HD to improve the present treatment of this disease.

The intervertebral disc is composed of an outer annulus fibrosus (AF) arranged in concentric lamellae surrounding an inner gelatinous nucleus pulposus (NP) (2). The disc is comprised mainly of the large proteoglycan aggrecan and type II collagen (6-9). The intervertebral disc, nourished only through diffusion from upper and lower endplates of vertebrae, lacks its own blood supply. Over a normal life-span, the total proteoglycan and water content in disc tissues is found to decrease, which may result in an increased susceptibility to herniation (10). HD is classically described as the protrusion of a degenerated disc into the spinal canal or neural foramina (11). This protrusion can result in compressed nerve tissue, depending upon the extent of the protrusion, and neovascularization of the HD from the spinal canal where there is an abundant blood supply. Surgical samples from patients with HD display granulation tissue with prominent macrophage and mild T-lymphocyte infiltration concomitant with the onset of neovascularization that is not observed in healthy intervertebral disc (12).

Interestingly, more than $50 \%$ of patients with back pain and sciatica have been reported to recover within 6 weeks (13). Recently, a study with magnetic resonance imaging (MRI) has shown that epidurally displaced HD tissues more commonly undergo a gradual decrease in the size of HD than those with minimal protrusion, and this decrease is accompanied by a reduction in associated pain and neurological symptoms (14). This tendency for natural resorption correlated with the accumulation of a contrast agent gadolinium-diethylene pentaacetic acid within the vascularized granulation tissues of the HD (15). Changes initiated by contact with infiltrating cells may thus contribute to the resorption process.

The matrix metalloproteinases (MMPs) are secreted $\mathrm{Zn}^{2+}$-dependent proteases that degrade the components of the extracellular matrix and are believed to play a role in tissue remodeling in a number of normal and pathological conditions. We examined previously the expression of MMP-3 (stromelysin-1, EC\#3.4.24.17) and MMP-7 (matrilysin, EC\#3.4.24.23) in human surgical samples of HD because these MMPs have been shown 
to cleave the cartilage proteoglycan aggrecan $(16,17)$. Immunohistological analysis revealed that infiltrated macrophages and chondrocyte-like cells expressed both MMP-3 and MMP-7 in the granulation tissues of HD (18-20). In contrast, discs obtained from patients who underwent discectomy for scoliosis or spinal cord tumors and who appeared normal on MRI showed no such upregulation. These data suggest that these MMPs may play an important role in removing matrix during the resorption process of $\mathrm{HD}$, similar to their proposed role in cartilage degradation in arthritic conditions (21-23). We hypothesize that these MMPs are upregulated when infiltrating macrophages encounter chondrocytes in the intervertebral disc. In this study, we used a coculture system of chondrocytes and macrophages in an effort to reproduce the acute phase of disc herniation in vitro. Using MMP-null mice, we have identified a specific role for MMP-3 in the resorption process of HD.

\section{Methods}

The experimental protocols were approved by the Institutional Animal Care and Use Committee, Vanderbilt University Medical Center.

Mice. Homozygous wild-type 129/Sv, MMP-3-null 129SvEv, and MMP-7-null 129SvEv mice were used in these studies. MMP-3-null and MMP-7-null mice were generated by homologous recombination in embryonic stem cells, as described $(24,25)$.

Preparation of macrophages. Activated murine macrophages were obtained by intraperitoneal administration of $2 \mathrm{~mL}$ PBS containing 3\% thioglycollate medium (Difco Laboratories, Detroit, Michigan, USA). For some experiments, red fluorescent cell linkers (PKH26; Sigma Chemical Co., St. Louis, Missouri, USA) were injected into the peritoneum 3 days after thioglycollate administration, according to the manufacturer's instructions. Cells were harvested by peritoneal lavage 4 days after thioglycollate treatment and cultured in DMEM (GIBCO BRL, Grand Island, New York, USA) supplemented with 10\% FBS (Atlanta Biologicals, Norcross, Georgia, USA), and $50 \mu \mathrm{g} / \mathrm{mL}$ gentamycin (GIBCO BRL). Chondrocyte cell culture. Rat RCJ 3.1C5.18 (C5.18) chondrocyte cells were generously provided by Jane Aubin (Department of Anatomy and Cell Biology, University of Toronto, Toronto, Canada) (26). C5.18 cells were resuspended in $1.2 \%$ low-viscosity alginate (Keltone; a gift from Monsanto Corp., St. Louis, Missouri, USA) in sterile sodium chloride at a concentration of $4 \times 10^{6}$ cells/mL of gel, then gradually expressed through a 21 gauge needle into a $102-\mathrm{mM}$ calcium chloride solution to promote gelling of the alginate. Gels were washed once with $0.15 \mathrm{M} \mathrm{NaCl}$ and 3 times with DMEM. The beads were cultured in a 1:1 mixture of DMEM and Ham's F-12 medium (GIBCO BRL) with 10\% FBS, 25 $\mu \mathrm{g} / \mathrm{mL}$ ascorbic acid (GIBCO BRL), and $50 \mu \mathrm{g} / \mathrm{mL}$ gentamycin in a humidified atmosphere of $5 \% \mathrm{CO}_{2}$ at $37^{\circ} \mathrm{C}$.

For primary chondrocyte cultures, intervertebral discs were obtained from the coccygeal spine after mice were sacrificed using carbon dioxide. The mouse tails were separated with a scalpel, and the skin and soft tissue were removed to expose the disc. The disc was sharply incised, and the immediate upper and lower coccygeal vertebrae were bent apart, forcing the gel-like nuclear material to protrude. The NP, observed as a gelatinous and semitransparent tissue, was obtained from all coccygeal discs. NP tissue was incubated in DMEM supplemented with $10 \% \mathrm{FBS}, 50 \mu \mathrm{g} / \mathrm{mL}$ gentamycin, and $0.05 \%$ collagenase (Sigma) overnight in a humidified atmosphere of $5 \% \mathrm{CO}_{2}$ at $37^{\circ} \mathrm{C}$. After digestion, the isolated chondrocytes were embedded in alginate beads as described above, except larger (30-mm diameter) beads were generated to measure macrophage infiltration.

Disc tissue cocultures. Coccygeal intervertebral disc tissues were obtained using a dissecting microscope after skin and soft tissues were removed. Whole intervertebral disc tissues (5 discs/35-mm dish) and PKH26labeled macrophages $\left(10^{6} / \mathrm{mL}\right)$ were cocultured in $5 \mathrm{~mL}$ Opti-MEM containing $50 \mu \mathrm{g} / \mathrm{mL}$ gentamycin for 2 days in a humidified environment of $5 \% \mathrm{CO}_{2}$ at $37^{\circ} \mathrm{C}$. After incubation, the medium was collected, and the disc tissues were embedded in OCT compound, snapfrozen in liquid nitrogen, and stored at $-80^{\circ} \mathrm{C}$.

To measure degradation of disc tissue quantitatively, intervertebral discs (1 disc per well of a 24-well plate; Corning-Costar, Cambridge, Massachusetts, USA) were cultured with or without peritoneal macrophages $\left(10^{6} / \mathrm{mL}\right)$ or 3 days in $1 \mathrm{~mL}$ Opti-MEM with $50 \mu \mathrm{g} / \mathrm{mL}$ gentamycin and $0.25 \mu \mathrm{g} / \mathrm{mL}$ Fungizone (GIBCO BRL). Before and after completion of incubation, samples were removed from the culture medium, patted briefly with sterile gauze to remove surface water, and their wet weight was measured with an autobalance (CAHN 28 automatic electrobalance; CAHN Instrument Company, Paramount, California, USA). Samples were then fixed overnight at $4{ }^{\circ} \mathrm{C}$ in freshly prepared $4 \%$ paraformaldehyde and were paraffin embedded. Sections were stained with $0.25 \%$ Safranin O as an indicator of proteoglycan content in tissue sections (27).

Cellular cocultures. Cell culture inserts $(8.0 \mu \mathrm{m}$; FALCON, Franklin Lakes, New Jersey, USA) were placed in each well of a 6-well plate (Corning-Costar Corp., Cambridge, Massachusetts, USA) thus forming transwell chambers. Primary chondrocytes or C5.18 cells in alginate beads were placed in the cell culture insert, and macrophages were placed on the bottom of each well. Cells cultured in DMEM containing 10\% FBS, 25 $\mu \mathrm{g} / \mathrm{mL}$ ascorbic acid, and $50 \mu \mathrm{g} / \mathrm{mL}$ gentamycin were either fixed overnight at $4^{\circ} \mathrm{C}$ in freshly prepared $4 \%$ paraformaldehyde and were paraffin embedded for immunohistochemical analysis, or were maintained in Opti-MEM for 2 to 4 days, then processed for determination of MMP mRNA and protein.

Immunobistological and histochemical staining. Paraffin or OCT-embedded sections were treated with $0.6 \%$ hydrogen peroxide in methanol to deplete endogenous peroxidase activity and incubated with the following murine monoclonal antibodies for 1 hour at room temperature: anti-human/mouse MMP-7 (1:100 dilution; 
Fuji Chemical, Toyama, Japan), anti-rat/mouse MMP-3 (1:500 dilution) (28), anti-human proteoglycan (1:500 dilution; Seikagaku Corporation, Tokyo, Japan), or antirat type II collagen (1:500 dilution; Chondrex, Seattle, Washington, USA). Mouse IgG (Sigma) was used as a negative control. Sections were then washed with $0.03 \%$ saponin (J.T. Baker Chemical Co, Phillipsburg, New Jersey, USA) in Tris-buffered saline (TBS; $0.15 \mathrm{M} \mathrm{NaCl}$, $0.01 \mathrm{M}$ Tris, $\mathrm{pH}$ 8.0) and incubated with biotinylated anti-mouse IgG (Histofine Immunohistologic Staining Kit; Nichirei, Tokyo, Japan) for 10 minutes, followed by incubation for 5 minutes with peroxidase-labeled streptavidin. Positive cells were visualized using True Blue peroxidase substrate, and sections were counterstained with contrast red (both from Kirkegaard and Perry Laboratories, Gaithersburg, Maryland, USA).

Western blotting. After 2 to 4 days in culture, serum-free cell culture medium was collected from single cultures and cocultures and total protein was quantitated using the bicinchoninic acid (Pierce Chemical Co., Rockford, Illinois, USA) or Bio-Rad (Bio-Rad Laboratories Inc., Hercules, California, USA) assays. Media samples were diluted 1:1 with Laemmli sample buffer, and $22.5 \mu \mathrm{g}$ of protein was subjected to SDS-PAGE (10\% gel) under reducing conditions; the proteins were transferred onto nitrocellulose membranes (Nitro ME; MSI, Westborough, Massachusetts, USA). Membranes were blocked with $5 \%$ nonfat dry milk in TBS containing $0.05 \%$ Tween-20 (TBS-T) for 1 hour followed by a 1:5000 dilution of anti-MMP-3 or anti-MMP-7 antibody. Rat MMP-3 (29) and recombinant mouse MMP-7 (30) expressed in baculovirus were used as positive controls. Membranes were then washed with TBS-T and incubated sequentially with biotinylated anti-mouse IgG (Vector Laboratories, Burlingame, California, USA) for 1 hour, washed again, and incubated with peroxidaseconjugated streptavidin (Jackson ImmunoResearch Laboratories Inc., West Grove, Pennsylvania, USA) for 1 hour. Immunoreactive proteins were identified by chemiluminescence with a solution of $2.5 \mathrm{mM}$ luminol (Sigma), $0.4 \mathrm{mM}$ p-coumaric acid (Sigma), and 0.02\% $\mathrm{H}_{2} \mathrm{O}_{2}$ in $50 \mathrm{mM}$ Tris (pH 6.5).

RT-PCR. Cells grown singly or under conditions of coculture were placed in sterile Eppendorf tubes, and total RNA was extracted using a modified guanidinium isothiocyanate procedure (31). One microgram of total RNA was incubated at $65^{\circ} \mathrm{C}$ for 10 minutes with oligo dT primers (Promega Corp., Madison, Wisconsin, USA) and then immediately chilled on ice. The cDNA synthesis reaction mixture of $5 \times$ buffer $(250 \mathrm{mM}$ Tris$\mathrm{HCl}, 375 \mathrm{mM} \mathrm{KCl}, 15 \mathrm{mM} \mathrm{MgCl} 2,50 \mathrm{mM}$ DTT; Promega), DTT, ribonuclease inhibitor, dNTPs, and MMLV-reverse transcriptase (all from Promega; used according to manufacturer's directions) was assembled on ice, and the reaction was allowed to proceed at $37^{\circ} \mathrm{C}$ for 1 hour. The cDNA synthesis reaction was terminated by boiling for 2 minutes and then chilling on ice. Five microliters of synthesized cDNA was used for each PCR. The cDNA mixture was supplemented with 10 pmol of $5^{\prime}$ and $3^{\prime}$ primers, $10 \times$ reaction buffer $(50 \mathrm{mM}$ Tris-HCl, $100 \mathrm{mM} \mathrm{NaCl}, 0.1 \mathrm{mM}$ EDTA, $1 \mathrm{mM}$ DTT, $50 \%$ glycerol, and $1.0 \%$ Triton-X-100; Promega), $\mathrm{MgCl}_{2}$ (1.5 mM), dNTPs $(0.25 \mathrm{mM})$, and Taq polymerase (1.5 U; Promega). A5 murine spindle cell carcinoma cell line-derived mRNA or MMP-7 plasmid was used as positive control for MMP-3 and MMP-7, respectively $(28,30)$. Sequence of the sense primers for MMP-3, MMP-7, and GAPDH were 5'-TTCTCCAGGATCTCTGAAGGAGAGG-3', 5'-TGGAGTGCCAGATGTTGCAG-3', and $5^{\prime}$-ACCACAGTCCATGCCATCAC-3', with antisense sequences of 5'-ATTTGGTGGGTACCACGAGGACATC-3', $5^{\prime}$-TTTCCATATAGCTTCTGAATGCCT- $3^{\prime}$, and 5'-TCCACCACCCTGTTGCTGTA- $3^{\prime}$, respectively. Products of 391 , 518, and 451 bp were predicted for MMP-3, MMP-7, and GAPDH, respectively. The MMP primers were designed to detect both mouse and rat MMP-3 and MMP-7 and not hybridize with other known MMPs. A 30-cycle PCR reaction was used, with each cycle consisting of denaturation at $94^{\circ} \mathrm{C}$ for 1 minute, annealing at $50^{\circ} \mathrm{C}$ for 1 minute, and primer extension at $72^{\circ} \mathrm{C}$ for 1 minute for MMP-3 or 2 minutes for MMP-7. RTPCR for GAPDH was performed at the same time and under the same conditions. Ten microliters of the reaction mixture was then subjected to electrophoresis in $2.0 \%$ agarose in $1 \times \mathrm{TBE}(89 \mathrm{mM}$ Tris borate and $2 \mathrm{mM}$ EDTA) buffer. The DNAs were visualized by ethidium bromide staining and ultraviolet illumination.

Migration assay and Boyden chamber checkerboard analysis. Migration activity of peritoneal macrophages was examined by a modified Boyden chamber assay with a 48-well chamber (Neuroprobe, Gaithersburg, Maryland, USA). The upper and lower chambers were separated with a polyvinylpyrrolidone-free polycarbonate filter with $8-\mu \mathrm{m}$ pores (Corning-Costar Corp.). Macrophages $\left(1.2 \times 10^{6}\right.$ cells $\left./ \mathrm{mL}\right)$ were suspended in coculture-conditioned medium or Opti-MEM and added to the upper wells. Conditioned media derived from cocultures of macrophages and either wild-type or MMP-3-null disc tissue diluted with Opti-MEM was added to the upper and/or lower chambers as indicated. Medium concentration was achieved with microconcentrator tubes (MICROCON, 3,000 molecular weight cut off; Amicon/Millipore, Beverly, Massachusetts, USA). Total volumes in each chamber were 27.5 $\mu \mathrm{L}$ in the lower and $50 \mu \mathrm{L}$ in the upper chamber. After incubation for 5 hours at $37^{\circ} \mathrm{C}$, the membrane was removed, washed with PBS, and nonmigrated cells on the upper surface of the membrane were removed. Cells were fixed in methanol for 5 minutes, stained with hematoxylin for 5 minutes, and mounted on glass slides. Cells on the lower surface were counted in 6 high-power fields $(\times 400)$ per group. An insignificant number of macrophages were observed in the lower chamber at this time point and were not included in the analysis. Statistical analysis of the data was performed using the Mann-Whitney test. A $P$ value less than 0.05 was considered significant. Each experiment was performed in triplicate and repeated at least twice. 


\section{Results}

MMP expression in an organ culture model of HD. Human HD specimens demonstrate marked macrophage infiltration and the expression of MMP-3 and MMP-7 in both chondrocytes and infiltrated inflammatory cells (18-20). To provide an organ culture model of HD, coccygeal intervertebral discs were dissected from adult mice. Peritoneal macrophages were recruited by thioglycollate administration, and isolated macrophages were placed in direct contact with the disc tissue and maintained in serum-free medium for 2 days. To determine if MMP expression in disc organ cocultures recapitulated that observed in human HD tissues, frozen sections were analyzed for murine MMP levels by immunohistological staining. Disc cells expressed detectable levels of MMP-3 and MMP-7 (Figure 1). Cultures of embedded disc cells in the absence of macrophages had no detectable MMP-3 or MMP-7 (data not shown). No immunohistologically positive cells were seen in control immunoglobulin-treated sections. These features were similar to the findings in human HD samples and indicate that the disc organ culture system is appropriate for the study of the role of MMP-3 and MMP7 in HD resorption.

To address the contributions of chondrocytes and macrophages independently, a cellular culture system was developed. Monolayer culture of chondrocytes does not support a differentiated phenotype as measured by the loss of aggrecan and type II collagen production and the onset of type I collagen synthesis (32). Thus, we turned to a 3-dimensional culture system in which a linear polysaccharide, alginate, was used to encapsulate cells by forming a gel in the presence of calcium (33). The use of this matrix to grow chondrocytes or disc cells has been demonstrated to maintain the differentiated phenotype of such cells $(34,35)$. Immortal C5.18 rat chondrocyte cells were embedded in alginate beads as described previously $(34,36)$. The cells formed lacunaelike structures, demonstrated extensive Safranin O staining, and expressed proteoglycan and type II collagen as determined by immunohistological staining (data not shown). Based on these results, encapsulated C5.18 cells adopted the phenotype of a differentiated chondrocyte.

MMP expression in cellular cocultures of chondrocytes and macrophages. The production of MMP-3 and MMP-7 by chondrocytes and macrophages cultured singly or cocultured in transwell chambers was analyzed by Western blotting of conditioned medium. Differentiated C5.18 cells in alginate beads produced low levels of MMP-3, but not MMP-7, at 2 and 4 days of culture (Figure 2, a and $\mathrm{b}$, lane $\mathrm{C}$ ). Isolated macrophages produced MMP-7 only during the initial culture period (Figure 2, a and b, lane $\mathrm{M}$ ). In contrast, media derived from coculture samples were strongly positive for MMP-3 and MMP-7 throughout the culture time (Figure 2, a and b, lane MC). Thus, the production of both MMP-3 and MMP-7 was strongly upregulated by the conditions of coculture.

To determine if the MMP-3 and MMP-7 protein produced in cocultures was synthesized by the chondrocytes or the macrophages, mRNA from chondrocytes (upper transwell chamber) or macrophages (lower transwell chamber) was analyzed using RT-PCR. Chondrocytes alone have barely detectable MMP-3 mRNA (Figure 3, lane C). However, MMP-3 mRNA was easily detected in the chondrocytes following coculture with macrophages and appeared to be abundantly expressed, although the RT-PCR technique used does not provide quantitative information (Figure 3, lane M-C). MMP-7 mRNA was not readily detectable in chondrocytes with or without coculture under the conditions used. The peritoneal macrophages produced MMP-3 and MMP-7 mRNA in both single and cocultured conditions (Figure 3). These results are consistent with the results of the Western analysis and indicate that MMP-3 was produced by both chondrocytes and macrophages in coculture conditions and that MMP-7 was produced predominantly by the macrophages.

Requirement for MMP-3 for disc degradation. To determine if chondrocyte MMP-3 or MMP-7 was required for resorption of disc tissues in the coculture model system, we examined the degradation of wild-type, MMP-3-null and MMP-7-null discs cocultured with peritoneal macrophages by measuring wet weight and Safranin O staining. Safranin O staining has been reported as a quantitative indicator of proteoglycan content in tissue sections (27). Wild-type and MMP7-null disc tissues cocultured with macrophages for 3 days had irregular surfaces and a flocculent appearance by macroscopic observation, whereas the surfaces of MMP-3-null cocultured discs, or either discs in the absence of macrophages, were distinctly defined (data not shown). Degradation of wild-type and MMP-7-/-

\section{Figure 1}

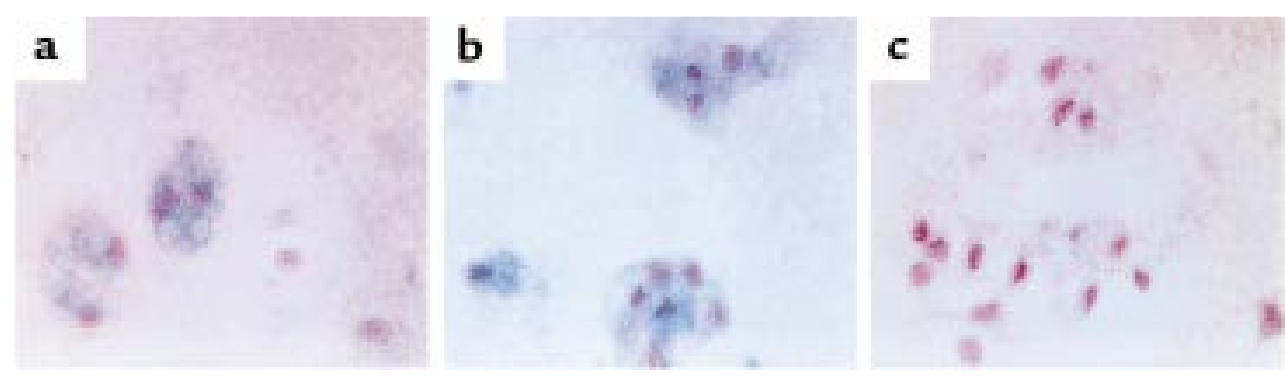

Immunohistochemical analysis of MMP-3 and MMP-7 in macrophage/disc cocultures. Frozen sections of murine coccygeal intervertebral discs cocultured with peritoneal macrophages were immunostained with anti-rat/mouse MMP-3 (a), anti- human/mouse MMP-7 (b), and control IgG (c) antibodies and visualized using True Blue peroxidase substrate. Counterstain is contrast red. $\times 400$. 
a

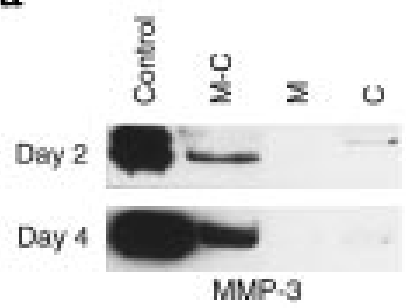

b

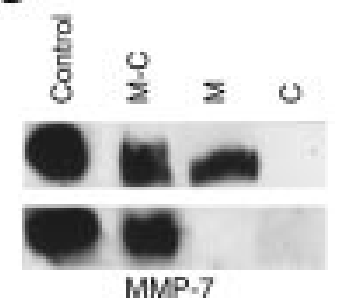

\section{Figure 2}

MMP-3 and MMP-7 protein expression in chondrocyte/macrophage cocultures. Western blotting analysis for MMP-3 (a) or MMP-7 (b) of media from peritoneal macrophages (M) and C5.18 cells grown singly (C) or in coculture (M-C). Recombinant rat MMP-3 (a, control) and baculovirus-produced murine MMP-7 (b, control) were used as positive controls.

disc tissue during coculture with macrophages was confirmed by a significant loss of Safranin O staining (Figure 4a) and a decrease in wet weight $(P<0.01$; Figure $4 \mathrm{~b})$ after 3 days of coculture. In contrast, MMP3 -null discs demonstrated no loss of wet weight and were spared from significant proteoglycan loss (Figure 4 , $a$ and $b$ ). These data demonstrated that disc-derived MMP-3 was required for the physical degradation of disc tissues cocultured with macrophages to occur.

To determine if MMP- 3 production by chondrocytes is sufficient for disc degradation, isolated discs were treated with the cytokine TNF- $\alpha$, a known inducer of MMP-3 expression in chondrocytes (37). TNF- $\alpha$ treatment resulted in a marked elevation in MMP-3 protein levels in disc-conditioned medium (Figure 4b, inset). However, there was no significant disc degradation as measured by the loss of wet weight (Figure 4b). Thus, the induction of chondrocyte MMP-3 levels by coculture with macrophages was necessary, but not sufficient, for matrix degradation in this model of HD resorption.

Requirement for chondrocyte MMP-3 for macrophage infiltration. The degradation and proteoglycan loss that occurred in wild-type disc tissue in disc/macrophage cocultures appeared to be accompanied by a marked increase in cellularity, suggesting that macrophage penetration of the disc tissue was a component of the resorption process. In contrast, MMP-3-null discs cocultured with macrophages lacked this cellularity and resembled discs that were not exposed to macrophages (Figure 4a). To determine if chondrocyte MMPs were affecting macrophage infiltration of disc tissue, coccygeal discs isolated from wild-type, MMP-3-null, and MMP-7-null mice were cocultured with peritoneal macrophages that had been prelabeled with PHK26 red fluorescent cell linkers, and frozen sections of tissues were analyzed for macrophage recruitment. The number of red fluorescent macrophages infiltrating into the outer AF layer, the inner NP layer, and the intermediate transitional zone of the disc organ cultures was quantitated. The results indicated that the infiltration of macrophages in MMP-3-null discs was strongly inhibited (Figure 5). In contrast to wild-type discs, where macrophages were observed to infiltrate all 3 layers of the disc, the majority of macrophages remained on the exterior surface of the MMP-3-null disc with no infiltration of the transitional zone or NP and with a more than $95 \%$ reduction in the number of cells entering the $\mathrm{AF}$. The macrophages appeared to penetrate the NP of MMP-7-null discs as efficiently as wild-type discs, although there was a slight and statistically insignificant reduction in the absolute numbers of macrophages in each zone. A similar lack of macrophage infiltration, compared with wild-type controls, was observed when MMP-3-null chondrocytes were embedded in a large alginate bead and infiltration into the center of the bead was quantitated (data not shown). Thus, chondrocyte MMP-3 appeared to be required for macrophage penetration irrespective of the chemical composition of the matrix that was infiltrated. These results suggest that chondrocyte MMP-3 activity may play a role in chondrocyte/macrophage communication through the release of macrophage chemoattractive factors.

Chondrocyte MMP-3 releases a macrophage chemotactic factor. To determine if MMP-3 played a role in the release of a macrophage chemoattractant, conditioned medium from single or cocultured chondrocytes was tested in a modified Boyden chamber migration assay. Conditioned medium from discs alone resulted in modest macrophage migration (Figure 6a). In contrast, medium from cocultures of chondrocytes and macrophages resulted in a 5.6-fold increase in macrophage migration. When medium was collected from cocultures of MMP-3-null chondrocytes and macrophages, migration was reduced to control levels. We conclude that MMP-3 production by chondrocytes in the presence of macrophages contributes to the generation of a factor that stimulates macrophage migration.

Stimulation of macrophage migration by a chondrocyte-released factor could involve enhancement of either directional migration (chemotaxis), increased random

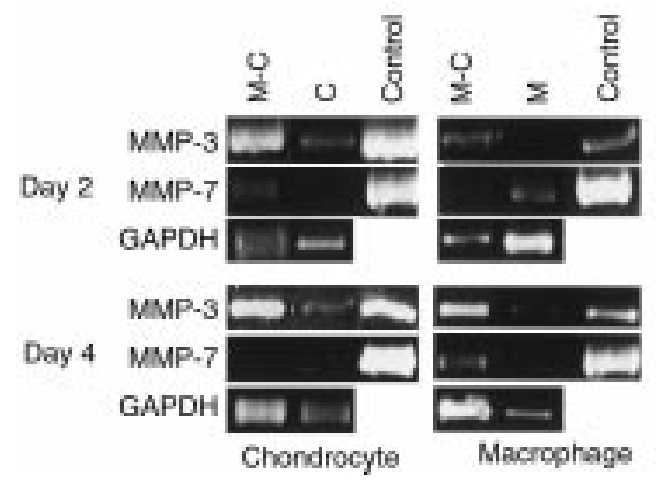

\section{Figure 3}

MMP-3 and MMP-7 mRNA from chondrocytes and macrophages in coculture. RT-PCR analysis of RNA isolated from C5.18 cells (C) and peritoneal macrophages $(\mathrm{M})$ cultured in transwells either alone or in coculture (M-C). A5 spindle cell carcinoma cell line-derived mRNA and MMP-7 plasmid DNA were used as positive controls (control). GAPDH transcripts were coamplified with the MMP transcripts and are depicted in the lower lanes of each panel. 
motility (chemokinesis), or a combination of both. To identify these activities, a modified Boyden chamber checkerboard analysis was performed. A concentration gradient in both the upper and lower chambers of transwell cultures was formed by diluting or concentrating conditioned medium from macrophages that were cocultured with either wild-type or MMP-3-null chondrocytes. Macrophage migration through an $8-\mu \mathrm{m}$ pore membrane was measured in response to this gradient. Chemokinetic activity, indicated by an increase in migration with increasing concentrations of medium in the upper or the upper and lower chambers, was observed at high concentrations of conditioned medium from macrophages cocultured with either wild-type or MMP-3-null discs. In contrast, chemotactic activity, indicated by increased migration in response to a concentration gradient, was observed only with conditioned medium from macrophage/wild-type disc cocultures. The values obtained with conditioned medium from macrophage/MMP-3-null disc cocultures were subtracted from those obtained with macrophage/wildtype discs to normalize for the presence of the background chemokinetic activity; the results are presented in Figure 6b. When conditioned medium was placed in the bottom chamber only, there was a marked, dosedependent enhancement in macrophage migration that peaked at 200\% medium (Figure 6b, 0\% conditioned medium in upper chamber). A decrease in migration characteristic of desensitization was observed at the highest concentration of conditioned medium in the lower chamber. A similar chemotactic response toward a concentration gradient was observed when macrophage/wild-type disc-conditioned medium at a concentration of $50 \%$ and $100 \%$ was placed in the upper chamber, although the increase in migration was not observed until the concentration in the lower chamber exceeded that in the upper chamber (Figure 6). These results indicate that the MMP-3-dependent factor has chemotactic activity as it fulfills the requirement of directional motility toward a gradient and desensitization of the effect at high factor concentrations.

\section{Discussion}

Spontaneous resorption of HD is commonly observed when there is substantial contact of the disc with the spinal canal, vascularization, and marked macrophage infiltration $(12,14,15)$. Enhanced expression of MMP-3 and MMP-7 in the cells of macrophage-infiltrated discs (18-20) suggested the hypothesis that MMP induction by infiltrating macrophages facilitated spontaneous $\mathrm{HD}$ resorption. Because human samples afforded the opportunity for correlative studies only, and no suitable animal model existed for HD, we devised organ and cell coculture systems to study the interaction of macrophage and disc chondrocytes in HD resorption. The simplest form of coculture involved the direct placement of isolated macrophages on intact intervertebral discs in culture medium. Marked infiltration of the macrophages and expression of MMP-3 and MMP-7 was observed, similar to that seen in human HD surgical samples. Discs underwent substantial loss of wet weight and proteoglycan content under these conditions, mimicking the HD resorption process. The coculture model was further refined to culture of activated peritoneal macrophages with alginate-encased chondrocytes, either directly or in a transwell system, so that it provided information on the cellular source of molecules involved in the resorption process, including MMP-3 and MMP-7. Although the coculture system may not recapitulate HD completely in that all cell types present in HD samples are not represented, we have demonstrated the utility of this model for identifying molecular components important for disc resorption, macrophage infiltration, and communication between these 2 major cell types.

The disc resorption studies indicated that disc-produced MMP-3, but not MMP-7, was required for the a

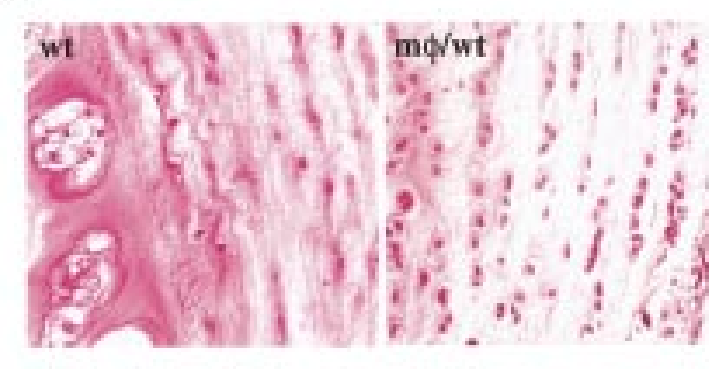

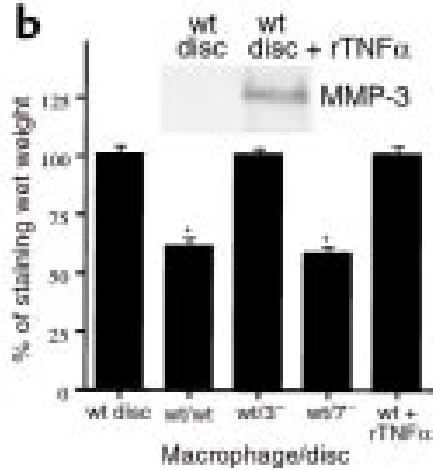

\section{Figure 4}

(a) Representative Safranin O-stained section of incubated whole disc tissue. Wild-type whole disc tissue cocultured with peritoneal macrophages $(\mathrm{m} \phi / \mathrm{wt}$ ) showed extensive reduction in staining compared with wild-type discs without macrophages (wt) or MMP-3-null whole disc tissue cocultured with macrophages $\left(\mathrm{m \phi} / 3^{-}\right) . \times 250$. (b) Quantitative analysis of wet weight loss in whole disc tissue from MMPnull organ cultures. Cocultures are indicated by the genotype of the mice donating macrophage/disc tissue. Wild-type discs were also treated with recombinant TNF $\alpha$ ( $r$ TNF $\alpha$ ) and assayed for MMP-3 protein by Western analysis (inset) and wet weight (wt $+r T N F \alpha)$. Data were analyzed with the Mann-Whitney test and were presented as mean $\pm \mathrm{SD}, n=7$ discs/group. ${ }^{*}$ Difference in wet weight between before and 3 days after incubation is significant $(P<0.01)$. wt, wild-type; $3^{-}$, MMP-3 null; $7^{-}$, MMP-7 null. 
loss of proteoglycan content and wet weight in the coculture system. The most straightforward interpretation of this result is that MMP-3 is required for proteolytic degradation of aggrecan, the major cartilage proteoglycan, and the loss of associated water molecules. There are several observations that argue against this interpretation. Although MMP-3 can cleave aggrecan at positions within the chondroitin sulfate-rich region (16), induction of MMP-3 protein in isolated discs by treatment with the cytokine TNF- $\alpha$ was not sufficient for disc degradation as measured by the loss of wet weight. In addition, MMP-3 activity is not required for the cartilage degradation that occurs in diseases such as rheumatoid arthritis, because MMP3-null mice display no reduction and even a slight enhancement of symptoms in a collagen-induced arthritis model system (24). An enzyme referred to as aggrecanase has been identified and determined to be a related but distinct metalloproteinase in the ADAMTS (a disintegrin and metalloproteinase-thrombospondin) family $(38,39)$. Although aggrecanase activity appears to be an important component of cartilage degradation associated with arthritis, it has been reported that it does not play a major role in aggrecan cleavage in intervertebral disc tissue (40). In our coculture model, the infiltration of macrophages correlated with disc resorption and was, in fact, required for disc degradation. Thus, although a contribution of disc aggrecanase cannot be ruled out, factors provided by infiltrating macrophages played a critical role in disc resorption in this model system. The role of chondrocyte MMP-3 in disc resorption thus appears to be primarily indirect and correlates with its requirement for macrophage infiltration as opposed to direct degradation of cartilage proteoglycan.

The macrophages appear to play at least 2 distinct roles in disc resorption. The transwell coculture experiments indicated that a soluble factor produced by macrophages induced MMP-3 protein and chondrocyte MMP-3 mRNA. Activated macrophages are known to generate proinflammatory cytokines such as TNF- $\alpha$ or IL-1 $\beta$ (41), which are potent inducers of various MMPs including MMP-3 (42, 43). Interestingly, we have determined using a neutralizing antibody approach that the presence of TNF- $\alpha$ in the coculture medium is required for chondrocyte MMP-3 expression (44). Macrophage function in disc resorption was not restored by the addition of TNF- $\alpha$, however, indicating that additional macrophage products are required for the loss of proteoglycan and wet weight. Our studies demonstrated a strong correlation between macrophage infiltration and disc resorption in that disc MMP-3, but not MMP-7, was required for both processes. Thus, physical degradation of the disc tissue is likely to be mediated by enzymatic activities elaborated by infiltrating macrophages. Macrophage MMPs are good candidates, because MMP-1 $(45,46)$, MMP-2 (45), MMP-3 (46), MMP-7 (47), MMP-9 (45), MMP-12 (48), and MMP-13 (49) are expressed by activated

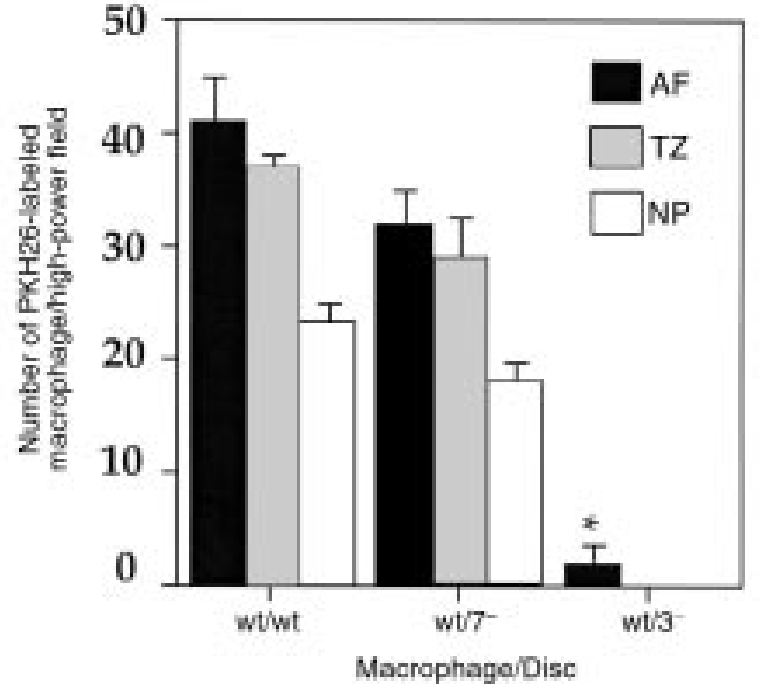

\section{Figure 5}

Quantitative analysis of macrophage infiltration in MMP-null organ cultures. A representative experiment showing the number of $\mathrm{PKH} 26$ labeled red fluorescent-infiltrated peritoneal macrophages in 3 highpower fields per zone of cocultured discs from wild-type (wt) and MMP-null mice (7-, MMP-7-null; 3-, MMP-3-null) is depicted. Cocultures are indicated by the genotype of the mice donating macrophages/disc tissue. Whole disc tissue was classified into 3 zones, NP (inner gelatinous region), AF (outer layer), and transition zone ( $T Z$; intermediate layer). Data were analyzed with the KruskalWallis test and were presented as mean $\pm \mathrm{SD}$. * Difference from wildtype is significant $(P<0.05)$.

macrophages, although we cannot rule out contributions by enzymes of other classes. We have evidence that neither MMP-3 nor MMP-7 production by macrophages is sufficient for disc resorption, because MMP-3-null macrophages and MMP-7-null macrophages supplemented with TNF- $\alpha$ effectively reduce disc wet weight in coculture (44). It is likely that a cascade of interacting proteases with complementary specificities for the matrix components of cartilage are involved in the resorption process of HD.

Because the macrophages were important contributors to disc resorption, we also examined the role of macrophage MMP-7 and MMP-3 in the coculture model of HD resorption (44). MMP-7 production by macrophages was required for the generation of TNF$\alpha$ and thus played a role in the communication between macrophages and chondrocytes. Macrophage MMP-3, in contrast, was dispensable for macrophage infiltration and for disc resorption. Because our results indicated that chondrocyte MMP-3 was required to generate signals important for the infiltration of macrophages, it is reasonable to ask if the lack of chondrocyte MMP-3 altered other macrophage responses important for disc resorption. The culturing of MMP3-null discs with macrophages resulted in a significant reduction in the production of MMP-3 by the macrophages (data not shown), but the ability of MMP-3-null macrophages to invade normally and induce disc resorption indicated that the lack of 
macrophage MMP-3 induction was unimportant to the resorption process. MMP-7 mRNA was produced by peritoneal macrophages in the presence or absence of chondrocytes, so the lack of MMP-3 in disc tissue is unlikely to alter the production of MMP-7 and generation of soluble TNF- $\alpha$. Thus, the lack of chondrocyte

a
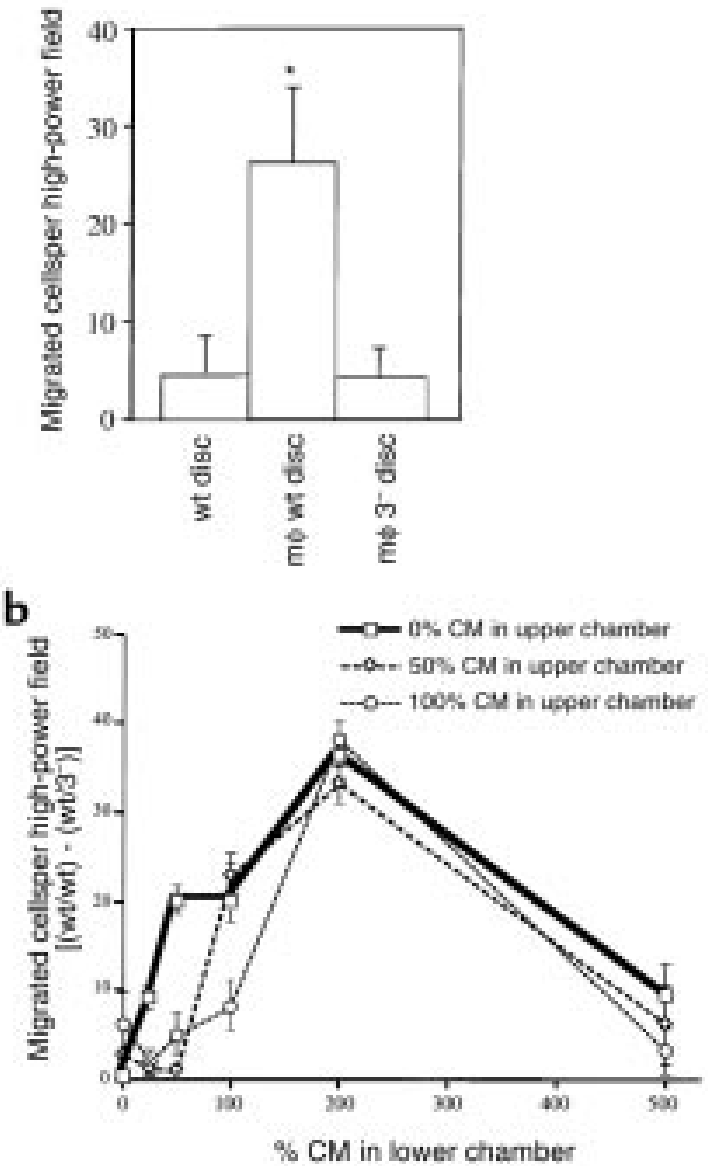

\section{Figure 6}

Chemotactic activity of macrophage/disc coculture-conditioned medium. Peritoneal macrophages were placed in the upper level of a Boyden chamber, and migration was quantified by counting the number of cells migrating to the lower side of the filter in 6 high-power fields $(\times 400)$ per well. (a) Medium in lower chamber was from wild-type (wt) or MMP-3-null ( $\left.3^{-}\right)$discs cocultured with or without macrophages $(\mathrm{m} \phi)$. * Significant migration $(P<0.05)$ compared with conditioned medium without macrophages. (b) Migration of wild-type macrophages toward macrophage/disc coculture media was analyzed using a modified Boyden chamber assay and diluting (<100\%) or concentrating ( $>100 \%$ ) conditioned medium (CM) from either wt macrophage/wt disc or wt macrophage/MMP-3-null disc cocultures. A checkerboard analysis of migration toward a concentration gradient was performed by placing increasing concentrations of coculture medium in the lower chamber with $0 \%$ (squares), $50 \%$ (diamonds), or $100 \%$ (circles) media in the upper chamber. Plotted are the average number of cells induced to migrate by wt/wt coculture medium with the MMP3 -independent background (number of cells induced to migrate by $w t / 3^{-}$medium) subtracted. Note that enhanced migration does not occur until the concentration of the medium in the lower chamber exceeds the concentration of the medium in the upper chamber; i.e., a concentration gradient is established.
MMP-3 did not appear to alter macrophage functions that were important for disc infiltration.

The most interesting observation from these studies is the role of chondrocyte MMP-3 in the generation of a chemotactic factor that induces macrophage infiltration. MMP-3-null discs did not allow macrophage infiltration in the organ coculture model, and isolated chondrocytes lacking MMP-3 did not allow macrophage infiltration of alginate beads. In addition, coculture medium from wild-type macrophages and MMP-3-null discs did not produce macrophage chemoattractants in the checkerboard chemoattraction assay. The nature of the MMP-3-dependent chemoattractant is not clear. Potential candidates include cell-surface chemokines such as monocyte chemotactic protein-1 (MCP-1). Chemokine MCP-1 is a potent macrophage chemoattractant that has been shown to be expressed by disc cells and infiltrated macrophages in human HD samples (12). Alternatively, MMP-3-mediated degradation of matrix components may release macrophage chemoattractants. For example, osteopontin is a glycosylated phosphoprotein secreted by chondrocytes that has been shown to be a macrophage chemoattractant (50). Interestingly, we have observed that MMP-3 cleavage of osteopontin increases its chemoattractant activity (unpublished data). In addition, we cannot rule out the possibility that MMP-3 inactivates an inhibitory protein that interferes with an endogenous chondrocyte chemoattractant. Irrespective of the molecular nature of the chemoattractant, this is, to our knowledge, the first example of a definitive role for MMP-3 in a chemoattractive process.

Our initial hypothesis was that MMP-3 and/or MMP7 would play a role in the degradation of cartilage proteoglycan during spontaneous HD resorption. The results of our studies indicated that chondrocyte MMP3, but not MMP-7, was required for disc resorption, but that the mechanism underlying this response was related to a role in cell/cell communication as opposed to a direct role on proteoglycan degradation. Several factors involved in the communication between macrophages and chondrocytes were identified using the coculture approach, although additional paracrine signals are likely to be uncovered. For example, TNF- $\alpha$ treatment of discs induced MMP-3, but was insufficient in itself to generate the chemoattractant (data not shown), suggesting that an additional macrophage factor cooperates with TNF- $\alpha$ to induce and/or release the chemoattractive activity. Our results reinforce a theme that has received increasing attention over the last several years regarding the importance of proteolytic regulation of biological processes. In addition to the classic examples of the complement and fibrinolysis cascades, proteosome-mediated degradation of signaling molecules and caspase regulation of programmed cell death are acknowledged as important regulatory mechanisms. There is increasing evidence that ectodomain shedding of cell-surface factors and their receptors may also regulate biological activity (51). The example of MMP-3 involvement in the generation of a macrophage 
chemoattractant and its requirement in HD resorption provides additional evidence for this premise.

\section{Acknowledgments}

The authors thank John Mudgett (Merck Research Laboratories) for MMP-3-null mice and Melodie Henderson for assistance with animal husbandry; Lucy Liaw, James Owen, and Diana Hulboy for technical advice; and Ann Richmond, Shinichi Osada, and Michihiko Wada for critical review of the manuscript. This work was supported by the Department of Orthopaedics and Rehabilitation, Vanderbilt University, Nashville, Tennessee and, in part, by the National Institutes of Health (grants CA-46843 and CA-60867 to L.M. Matrisian).

1. Frymoyer,J.W., and Cats-Baril, W.L. 1991. An overview of the incidence and costs of low back pain. Orthop. Clin. N. Am. 22:263-271.

2. Borenstein, D. 1996. Epidemiology, etiology, diagnostic evaluation, and treatment of low back pain. Curr. Opin. Rheumatol. 8:124-129.

3. Cypress, B.K. 1983. Characteristics of physician visits for back symptoms: a national perspective. Am. J. Public Health. 73:389-395.

4. Deyo, R.A., and Tsui-wu, Y.-J. 1987. Descriptive epidemiology of low-back pain and its related medical care in the united states. Spine. 12:264-268.

5. Anderson, G.B.J., and Weinstein, J.N. 1996. Disc herniation [editorial]. Spine. 21(Suppl. 24):1S.

6. Roberts, S., Caterson, B., Evans, H., and Eisenstein, S.M. 1994. Proteoglycan components of the intervertebral disc and cartilage endplate: an immunolocalization study of animal and human tissues. Histochem. J. 26:402-411.

7. Eyre, D.R., and Muir, H. 1976. Types I and II collagens in intervertebral disc: interchanging radial distributions in annulus fibrosus. Biochem. $\mathrm{J}$. 157:267-270.

8. Eyre, D.R., and Muir, H. 1977. Quantitative analysis of type I and II collagens in human intervertebral discs at various ages. Biochim. Biophys. Acta. 492:29-42.

9. Nerlich, A.G., Boos, N., Wiest, I., and Aebi, M. 1998. Immunolocalization of major interstitial collagen types in human lumbar intervertebral discs of various ages. Virchows Arch. 432:67-76.

10. Lipson, S.J., and Muir, H. 1981. Experimental intervertebral disc degeneration: morphologic and proteoglycan changes over time. Arthritis Rheum. 24:12-21.

11. Wisneski, R.J., Garfin, S.R., and Rothman, R.H. 1992. Lumbar disc disease. In The spine. 3rd edition. R.H. Rothman and F.A. Simeone, editors. W.B. Saunders Co. Philadelphia, PA. 671.

12. Haro, H., et al. 1996. Upregulated expression of chemokines in herniated nucleus pulposus resorption. Spine. 21:647-652.

13. Frymoyer, J.W. 1988. Back pain and sciatica. N. Engl. J. Med. 318:291-300.

14. Komori, H., et al. 1996. The natural history of herniated nucleus pulposus with radiculopathy. Spine. 21:225-229.

15. Komori, H., et al. 1998. Contrast-enhanced magnetic resonance imaging in conservative management of lumbar disc herniation. Spine. 23:67-73.

16. Bonassar, L.J., et al. 1995. Changes in cartilage composition and physical properties due to stromelysin degradation. Arthritis Rheum. 38:173-183.

17. Fosang, A.J., et al. 1992. The interglobular domain of cartilage aggrecan is cleaved by PUMP, gelatinases, and cathepsin B. J. Biol. Chem. 267:19470-19474.

18. Haro, H., Shinomiya, K., Murakami, S., and Spengler, D.M. 1999. Up-regulated expression of matrilysin and neutrophil collagenase in human herniated discs. J. Spinal Disord. 13:245-249.

19. Kanemoto, M., Hukuda, S., Komiya, Y., Katsuura, A., and Nishioka, J. 1996 Immunohistochemical study of matrix metalloproteinase-3 and tissue inhibitor of metalloproteinase-1 in human intervertebral discs. Spine. 21:1-8.

20. Haro, H., Murakami, S., Komori, H., Okawa, A., and Shinomiya, K. 1997. Chemonucleolysis with human stromelysin-1. Spine. 22:1098-1104.

21. Ohta, S., et al. 1998. Expression of matrix metalloproteinase 7 (matrilysin) in human osteoarthritic cartilage. Lab. Invest. 78:79-87.

22. Okada, Y., Takeuchi, N., Tomita, K., Nakanishi, I., and Nagase, H. 1989. Immunolocalisation of matrix metalloproteinase 3 (stromelysin) in rheumatoid synovioblasts (B cells): correlation with rheumatoid arthritis. Ann. Rheum. Dis. 48:645-653.

23. Okada, Y., et al. 1992. Localization of matrix metalloproteinase 3 (stromelysin) in osteoarthritic cartilage and synovium. Lab. Invest. 66:1069-1073.

24. Mudgett, J.S., et al. 1998. Susceptibility of stromelysin 1-deficient mice to collagen-induced arthritis and cartilage destruction. Arthritis Rheum. 41:110-121.
25. Wilson, C.L., Heppner, K.J., Labosky, P.A., Hogan, B.L.M., and Matrisian, L.M. 1997. Intestinal tumorigenesis is suppressed in mice lacking the metalloproteinase matrilysin. Proc. Natl. Acad. Sci. USA. 94:1402-1407.

26. Grigoriadis, A.E., Aubin, J.E., and Heersche, J.N. 1989. Effects of dexamethasone and vitamin D3 on cartilage differentiation in a clonal chondrogenic cell population. Endocrinology. 125:2103-2110.

27. Laurence, R. 1971. Chemical basis for the histological use of Safranin O in the study of articular cartilage. J. Bone Joint Surg. Am. 53:69-82.

28. Wright, J.H., et al. 1994. A switch from stromal to tumor cell expression of stromelysin-1 mRNA associated with the conversion of squamous to spindle carcinomas during mouse skin tumor progression. Mol. Carcinog. 10:207-215

29. Matrisian, L.M., et al. 1986. The mRNA coding for the secreted protease transin is expressed more abundantly in malignant than in benign tumors. Proc. Natl. Acad. Sci. USA. 83:9413-9417.

30. Wilson, C.L., Heppner, K.J., Rudolph, L.A., and Matrisian, L.M. 1995. The metalloproteinase matrilysin is preferentially expressed by epithelial cells in a tissue-restricted pattern in the mouse. Mol. Biol. Cell. 6:851-869.

31. Chomczynski, P., and Sacchi, N. 1987. Single-step method of RNA isolation by acid guanidinium thiocyanate-phenol-chloroform extraction. Anal. Biochem. 162:156-159.

32. Benya, P.D., and Shaffer, J.D. 1982. Dedifferentiated chondrocytes reexpress the differentiated collagen phenotype when cultured in agarose gels. Cell. 30:215-224.

33. Guo, J., Jourdian, G.W., and MacCallum, D.K. 1989. Culture and growth characteristics of chondrocytes encapsulated in alginate beads. Connect. Tissue Res. 19:277-297.

34. Häuselmann, H.J., et al. 1994. Phenotypic stability of bovine articular chondrocytes after long-term culture in alginate beads. J. Cell Sci. 107:17-27.

35. Chiba, K., Andersson, G.B.J., Masuda, K., and Thonar, E.J. 1997. Metabolism of the extracellular matrix formed by intervertebral disc cells cultured in alginate. Spine. 22:2885-2893.

36. Lowe, G.N., et al. 1996. Effects of prostaglandins on deoxyribonucleic acid and aggrecan synthesis in the RCJ 3.1C5.18 chondrocyte cell line. Endocrinology. 137:2208-2216.

37. Horton, W.E.J., Udo, I., Precht, P., Balakir, R., and Hasty, K. 1998. Cytokine inducible matrix metalloproteinase expression in immortalized rat chondrocytes is independent of nitric oxide stimulation. In Vitro Cell. Dev. Biol. 34:378-384

38. Fosang, A.J., Last, K., and Maciewicz, R.A. 1996. Aggrecan is degraded by matrix metalloproteinases in human arthritis. Evidence that matrix metalloproteinase and aggrecanase activities can be independent. J. Clin. Invest. 98:2292-2299.

39. Tortorella, M.D., et al. 1999. Purification and cloning of aggrecanase-1: a member of the ADAMTS family of proteins. Science. 284:1664-1666.

40. Sztrolovics, R., Alini, M., Roughley, P.J., and Mort, J.S. 1997. Aggrecan degradation in human intervertebral disc and articular cartilage. Biochem. J. 326:235-241.

41. Baumann, H., and Gauldie, J. 1994. The acute phase response. Immunol. Today. 15:74-80.

42. MacNaul, K.L., Chartrain, N., Lark, M., Tocci, M.J., and Hutchinson, N.I. 1990. Discoordinate expression of stromelysin, collagenase, and tissue inhibitor of metalloproteinases-1 in theumatoid synovial fibroblasts. Synergistic effects of interleukin-1 and tumor necrosis factor-alpha on stromelysin expression. J. Biol. Chem. 265:17238-17245.

43. Ganu, V.S., et al. 1994. Biochemical and molecular characterization of stromelysin synthesized by human osteoarthritic chondrocytes stimulated with recombinant human interleukin-1. Clin. Exp. Rheumatol. 12:489-496.

44. Haro, H., et al. Matrix metalloproteinase-7-dependent release of tumor necrosis tumor necrosis factor- $\alpha$ in a model of herniated disc resorption. J. Clin. Invest. 105:143-150.

45. Nakashima, Y., et al. 1998. Induction of matrix metalloproteinase expression in human macrophages by orthopaedics particulate debris in vitro. $J$. Bone Joint Surg. Br. 80: 694-700.

46. Mach, F., Schönbeck, U., Bonnefoy, J.-Y., Pober, J.S., and Libby, P. 1997. Activation of monocyte/macrophage functions related to acute atheroma complication by ligation of CD40: induction of collagenase, stromelysin, and tissue factor. Circulation. 96:396-399.

47. Busiek, D.F., et al. 1992. The matrix metalloproteinase matrilysin (PUMP) is expressed in developing human mononuclear phagocytes. J. Biol. Chem. 267:9087-9092.

48. Shapiro, S.D., Kobayashi, D., and Ley, T. 1993. Cloning and characterization of a unique elastolytic metalloproteinase produced by human alveolar macrophages. J. Biol. Chem. 268:23824-23829.

49. Lindy, O., et al. 1997. Matrix metalloproteinase 13 (collagenase 3) in human rheumatoid synovium. Arthritis Rheum. 40:1391-1399.

50. Denhardt, D.T., and Guo, X. 1993. Osteopontin: a protein with diverse functions. FASEB J. 7:1475-1482.

51. Werb, Z. 1997. ECM and cell surface proteolysis: regulating cellular ecology. Cell. 91:439-442. 